\title{
EMISSÕES DE POLUENTES ATMOSFÉRICOS EM CONDIÇÕES REAIS DE PAVIMENTAÇÃO ASFÁLTICA: MATERIAL PARTICULADO, BLACK CARBON E HIDROCARBONETOS POLICÍCLICOS AROMÁTICOS
}

\author{
Victor P. Piracellia, Ismael R. Amadora, Fabio C. Sabino ${ }^{a}$, Jurandir P. Pinto ${ }^{a}$, Carlos R. Silva Jr. ${ }^{a}$ e Maria Cristina Solci ${ }^{\mathrm{a} *}$, ,(1) \\ aDepartamento de Química, Universidade Estadual de Londrina, 86057-970 Londrina - PR, Brasil
}

Recebido em 29/08/2019; aceito em 21/01/2020; publicado na web em 03/04/2020

\begin{abstract}
AIR POLLUTANTS EMISSIONS IN REAL CONDITIONS OF ASPHALT PAVING. PARTICULATE MATTER (PM), BLACK CARBON (BC) AND POLYCYCLIC AROMATIC HYDROCARBONS (PAHS). Abstract: Intensive use of asphalt generates pollutants into the atmosphere which are harmful to the environment and worrying for occupational health. The application of asphalt products leads to emissions of significant concentrations of polycyclic aromatic hydrocarbons (PAHs) and particulate matter (PM) to the atmosphere. This paper aims to assess the pollutant emissions into the atmosphere in real conditions of asphalt paving through sampling and quantification of PM, Black Carbon (BC) and PAHs in fine particulate fraction and PAHs in gas phase. Concentration intervals of $\mathrm{PM}_{1.0}, \mathrm{PM}_{2.5}, \mathrm{BC}_{1.0}$ and $\mathrm{BC}_{2.5}$ found during a highway reconstruction in Londrina/PR were respectively 5.6-20.5; 6.3-24.2; 2.0-6.8 and 1.8-6.5 $\mu \mathrm{g} \mathrm{m}^{-3}$. During asphalt surfacing at the same city's central bus station, the concentration intervals were 15.1-75.1; 19.7-150; 5.1-14.6 and 4.9-16.2 $\mu \mathrm{g} \mathrm{m}^{-3}$ for $\mathrm{PM}_{1.0}, \mathrm{PM}_{2.5}, \mathrm{BC}_{1.0}$ and $\mathrm{BC}_{2.5}$, respectively. PAHs in the gas and PM fractions were found in both situations with higher concentrations in the gas phase. The results of this work can provide useful information to society in order to improve practices and promote lower environmental impact and health protection.
\end{abstract}

Keywords: atmospheric pollution; paving; real-word emission.

\section{INTRODUÇÃO}

A pavimentação asfáltica é a principal forma de revestimento de vias de rodagem. $\mathrm{O}$ uso intensivo de asfalto gera poluentes para atmosfera ao longo dos processos de extração, da destilação do petróleo, da estocagem, do transporte, da mistura com agregados até sua aplicação. ${ }^{1,2}$ Asfalto é um resíduo de petróleo composto por uma mistura complexa de compostos alifáticos, aromáticos e heterocíclicos. Milhões de toneladas de asfalto são produzidas anualmente e utilizadas em indústrias de pavimentação.,4

Quando o asfalto é aquecido, há a geração de vapores. Com o resfriamento destes vapores, ocorre a condensação e formação de fumos provenientes predominantemente dos componentes mais voláteis do asfalto. Sendo assim, esses fumos possuem propriedades químicas e toxicológicas distintas do asfalto. Como os componentes dos vapores não são condensados instantaneamente, emissões poluentes para a atmosfera provenientes da aplicação do asfalto apresentam-se tanto na fase particulada como na fase gasosa. ${ }^{3,4}$

Estima-se que o asfalto cru possua uma ampla classe de hidrocarbonetos policíclicos aromáticos (HPA). Em análise da composição de HPA em ligante asfáltico de petróleo, os de maior massa molar (4 - 6 anéis) apresentaram maior concentração em comparação com os de menor massa ( $2-3$ anéis). ${ }^{5}$ No preparo industrial, os fumos gerados do asfalto possuem predominantemente HPA de baixa massa molar (2 - 3 anéis). ${ }^{2}$ Em condições de pavimentação simuladas em laboratório, a emissão de HPA também é predominante entre os mais leves (3 - 4 anéis). ${ }^{6}$ Por caracterização química da emissão de óleo asfáltico de petróleo, fenantreno (FEN) é o HPA emitido em maior concentração e os com mais de 4 anéis não foram detectados. ${ }^{7}$

Coletas de fumos e vapores de asfalto gerados por simulação em laboratório ou em tanques de armazenamento são mais práticas em comparação com amostragens em tempo real em locais de trabalho. Contudo, é difícil reproduzir em laboratório as mesmas condições de uma coleta de campo real, tanto pela variação na composição química

*e-mail: solci@uel.br dos fumos e vapores gerados, quanto por fatores como temperatura, tempo de aquecimento e taxa de aplicação. ${ }^{8}$

Estudos de fumo de asfalto expostos à pele de ratos apresentaram efeito mutagênico e causaram câncer de pele. Por investigação epidemiológica em pavimentação asfáltica, concluiu-se que há risco de trabalhadores da área adquirirem câncer de pulmão, estômago, pele e leucemia. ${ }^{6}$ De acordo com o Instituto Nacional de Saúde e Segurança do Trabalho dos Estados Unidos, sobre os efeitos à saúde por exposição ocupacional em asfaltamento: "A exposição de trabalhadores ao fumo de asfalto causa irritação nos olhos, nariz e garganta. Conclusões sobre efeitos crônicos como bronquite e câncer de pulmão não podem ser tomadas até o momento, no entanto, o risco não pode ser descartado". ${ }^{9}$ O potencial carcinogênico e outros sintomas provocados pela exposição ao asfalto são intensamente estudados. ${ }^{8,10,11}$ Contudo, a atribuição destes sintomas exclusivamente ao asfalto não é clara devido ao baixo número de casos, pouco tempo de exposição e à exposição simultânea a outros poluentes como: fumaça de cigarro, emissões veiculares, asbestos, etc. ${ }^{8}$

\section{Material Particulado (MP) e Black Carbon (BC)}

O aerossol atmosférico é composto por pequenas partículas, tanto sólidas quanto líquidas, que estão temporariamente suspensas no ar. Essas partículas são denominadas de material particulado (MP). A principal forma de classificação do MP é pelo diâmetro aerodinâmico médio da partícula. As partículas grossas possuem diâmetros entre 10 e 2,5 $\mu \mathrm{m}\left(\mathrm{MP}_{10}\right.$ e $\left.\mathrm{MP}_{2,5}\right)$ e as partículas finas iguais e inferiores a $2,5 \mu \mathrm{m}\left(\leq \mathrm{MP}_{2,5}\right)$. Dependendo do tamanho, as partículas podem atingir diferentes regiões do sistema respiratório e provocar danos como irritação, inflamações e até câncer de pulmão. As partículas do MP são individualmente invisíveis a olho nu, contudo, em concentrações elevadas, formam uma névoa que restringe a visibilidade e apresentam potenciais impactos ambientais incluindo efeitos no clima. ${ }^{12-14}$

Uma fração do MP de base orgânica é chamada de Black Carbon (BC). O termo "Black" refere-se a propriedades ópticas devido à alta capacidade de absorção de luz visível. As partículas têm aparência 
escura forte e variam em tamanho, mas geralmente são predominantes no MP fino. ${ }^{15,16}$

Distinto de outras formas de carbono, BC apresenta a combinação das seguintes propriedades físicas: forte absorção em $550 \mathrm{~nm}$ $\left(5 \mathrm{~m}^{2} \mathrm{~g}^{-1}\right)$, é refratário $\left(\mathrm{T}_{\text {vap }} \approx 4000 \mathrm{~K}\right)$, insolúvel em água e em solventes orgânicos e possui morfologia de pequenas esferas carbônicas agregadas. ${ }^{16}$ Por imagens de microscopia eletrônica de transmissão é possível distinguir nas esferas camadas grafíticas enrugadas formando uma "casca" ao redor de um núcleo amorfo. ${ }^{17} \mathrm{BC}$ age como forte sorvente para compostos orgânicos persistentes, incluindo HPA. ${ }^{15}$ Apesar dos efeitos diretos do BC sobre a luz e o albedo, o BC não faz parte dos padrões ambientais de qualidade de ar.

\section{Hidrocarbonetos policíclicos aromáticos (HPA)}

Os HPA são substâncias semivoláteis em condições atmosféricas sendo que os de menor massa molar (até 4 anéis) encontram-se predominantemente na fase gasosa, enquanto os de maior massa molar estão na fase particulada. ${ }^{18,19}$ Os HPA são formados principalmente pela queima de combustíveis fósseis e madeira e durante processos que utilizam derivados de petróleo. ${ }^{20}$ Nos processos de emissão de HPA a partir de combustão a baixas temperaturas há formação de HPA de baixa massa molar. Durante a combustão em motores de veículos a temperatura é elevada, os compostos orgânicos têm tendência para formação de radicais reativos produzindo HPA com maior massa molar. ${ }^{21}$ Nos derivados asfálticos a porcentagem de aromáticos é predominante entre os hidrocarbonetos, podendo chegar a mais de $90 \%$ dependendo do tipo de asfalto. ${ }^{5,22}$ Os HPA são considerados tóxicos, carcinogênicos ou de grande potencial carcinogênico. ${ }^{20,23}$

\section{Legislação e saúde}

Os HPA afetam a saúde das pessoas. Conforme várias agências internacionais como os Centros de Controle e Prevenção de Doenças (Centers for Disease Control and Prevention - CDC), o naftaleno é irritante para os olhos e vias respiratórias e vários HPA são considerados causadores de câncer. ${ }^{24}$ De acordo com a Agência Internacional de Pesquisa sobre o Câncer (International Agency for Research on Cancer - IARC), o benzo(a)pireno é considerado carcinogêneo humano (grupo 1), benzo(a)antraceno e criseno são considerados possivelmente cancerígenos para humanos (grupo 2B) como também outros HPA encontrados neste trabalho são classificados como não carcinogênicos (grupo 3). ${ }^{25}$ A Agência de Proteção Ambiental dos Estados Unidos (United States Environmental Protection Agency - US-EPA) atribui valores potenciais de 1,0 a 0,001 para os HPA sendo 1,0 para benzo(a)pireno e dibenzo(a,h)antraceno, 0,1 para benzo(a)antraceno, benzo(b)fluoranteno e indeno(1,2,3-cd)pireno, 0,01 para benzo $(\mathrm{k})$ fluoranteno e 0,001 para criseno. Esses potenciais foram obtidos a partir de carcinogenicidade observada pela aplicação de HPA em pele de ratos. ${ }^{26} \mathrm{~A}$ concentração de risco estimada pela US-EPA para inalação de benzo(a)pireno foi de $2 \mathrm{ng} \mathrm{m}^{-3}$, no entanto, o benzo(a)antraceno e dibenzo(a,h)antraceno foram avaliados e valores de risco ainda não estão disponíveis. ${ }^{27-29}$ A Organização Mundial da Saúde (World Health Organization - WHO) baseada em dados epidemiológicos indicou que o benzo(a)pireno na concentração de $1,2 \mathrm{ng} \mathrm{m}^{-3}$ no ar produz um risco de câncer de $1 / 10000 .{ }^{30}$

Além do potencial carcinogênico individual, o Instituto Nacional para Segurança e Saúde Ocupacional (National Institute for Occupational Safety and Health - NIOSH) considera o nível de exposição de $100 \mathrm{ng} \mathrm{m}^{-3}$ a voláteis de petróleo para trabalhadores em 8 horas de turno. A Administração de Segurança de Saúde Ocupacional (Occupational Safety and Health Administration) e a Conferência Americana de Higienistas Industriais Governamentais (American Conference of Governmental Industrial Hygienists) consideram o limite de exposição no ambiente de trabalho de $200 \mathrm{ng} \mathrm{m}^{-3}$ para compostos de petróleo solúveis em benzeno. ${ }^{31}$

\section{Razões de diagnóstico (RD)}

Alguns HPA identificados no ambiente são sugeridos como indicadores de certas fontes de emissão. Cada processo de emissão produz HPA em proporções geralmente constantes sendo possível identificar HPA característicos como marcadores ou assinaturas de fonte de emissão. Os HPA pirolíticos (ou pirogênicos) apresentam maior distribuição de massas molares, enquanto os HPA de petróleo (ou petrogênicos) têm predominância de menor massa molar. Para auxiliar na identificação de fontes de emissão são utilizadas razões de diagnóstico (RD) entre alguns HPA.

Considerando que, no Brasil, cerca de $95 \%$ das estradas pavimentadas são de revestimento asfáltico, este trabalho teve como objetivo avaliar as emissões de poluentes para a atmosfera em condições reais de pavimentação através da amostragem e quantificação de MP, BC e HPA na fração particulada fina, assim como HPA na fase gasosa. As amostragens ocorreram durante as obras de duplicação da uma rodovia intermunicipal e do recapeamento asfáltico no terminal de ônibus urbano de Londrina/PR. Serão apresentadas as razões de diagnóstico entre pares de HPA com o objetivo de caracterizar as assinaturas destas fontes de emissão.

\section{PARTE EXPERIMENTAL}

\section{Locais de amostragem}

Duas campanhas de amostragem foram realizadas durante obras de pavimentação asfáltica. A primeira campanha ocorreu durante obras na rodovia intermunicipal PR-445 em Londrina, em local aberto, especificamente em $23,330328^{\circ} \mathrm{S}$ e $51,187035^{\circ} \mathrm{O}$. Os amostradores foram posicionados à $3 \mathrm{~m}$ da marginal à rodovia, na altura de $3 \mathrm{~m}$ (Figura 1). Nos dias de coleta, ocorreram as etapas de terraplanagem e compactação do lado da pista próximo aos amostradores e imprimação (aplicação de camada selante de asfalto diluído de petróleo) do lado oposto. A imprimação ocorreu pelo menos em cinco dos dias de coleta, incluindo o primeiro e o último dia. Durante a obra, o tráfego da rodovia (misto) foi alocado às ruas marginais.

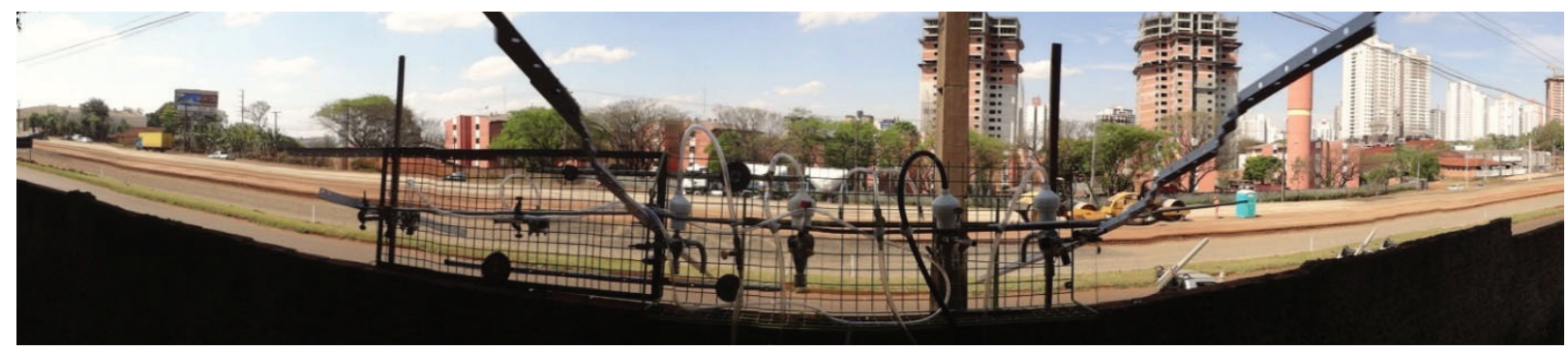

Figura 1. Amostradores instalados às margens da rodovia PR-445 durante obras de duplicação em Londrina 
Dados meteorológicos do período de amostragem foram obtidos do SIMEPAR (Sistema Meteorológico do Paraná).

A segunda campanha foi realizada no piso inferior do terminal de ônibus urbano localizado no centro de Londrina $\left(23,307870^{\circ} \mathrm{S}\right.$ e $51,161199^{\circ} \mathrm{O}$ ). $\mathrm{O}$ asfalto do piso inferior (área de $2875 \mathrm{~m}^{2}$ ) estava em reforma com remoção e substituição do revestimento asfáltico. O local é semiaberto, formado por paredes de $5 \mathrm{~m}$ de altura com aberturas de 2 e $3 \mathrm{~m}$ de altura nas laterais e na frente do prédio, respectivamente. Durante as obras, as linhas de ônibus que circulam no local foram transferidas para o piso superior. Os amostradores foram posicionados à $4 \mathrm{~m}$ de altura, à $3 \mathrm{~m}$ de uma das laterais do prédio. As amostragens tiveram início após a remoção do asfalto antigo. As etapas de imprimação e aplicação de massa asfáltica compreenderam os 3 primeiros dias de coleta. Após o $4^{\circ}$ dia, já havia tráfego de ônibus no local.

As campanhas foram realizadas no período de 20 a 29 de setembro de 2013 na PR-445 e no terminal durante 03 e 11 de abril de 2014, com amostragens de 24 horas. Para coleta de HPA na fase gasosa foram empregados, em duplicata, cartuchos de vidro recheados com duas sessões separadas em série de adsorvente polimérico AMBERLITE ${ }^{\circledR}$ XAD4 (800 e 200 mg) (Sigma-Aldrich ${ }^{\circledast}$, EUA) à vazão de $120 \mathrm{~L} \mathrm{~h}^{-1}$. A fase particulada foi coletada na fração fina $\left(\mathrm{MP}_{2,5}\right.$ e $\left.\mathrm{MP}_{1,0}\right)$ na $1^{\mathrm{a}}$ campanha e na $2^{\text {a }}$ campanha, sobre filtros de fibra de vidro de $47 \mathrm{~mm}$ de diâmetro (Sartorius ${ }^{\circledR}$, Alemanha). Ciclones URG ${ }^{\circledR}$ (Estados Unidos) à vazão de $1002 \mathrm{~L} \mathrm{~h}^{-1}$ foram empregados para coleta de $\mathrm{MP}_{2,5}$ e $\mathrm{MP}_{1,0}$. Brancos de campo dos cartuchos e filtros foram previamente selecionados. ${ }^{32}$ Foram utilizadas bombas de diafragma e as vazões foram mantidas através de válvulas de agulha. As vazões foram conferidas diariamente utilizando rotâmetro de esfera (Fischer \& Porter GmbH, Alemanha).

Após as amostragens, os cartuchos foram vedados e transportados ao laboratório onde foram mantidos sob refrigeração até extração. Os filtros foram armazenados em placas de Petri e transportados ao laboratório e foram mantidos em sala de ambientação até os procedimentos de análise. O transporte ao laboratório foi feito em caixa térmica e sob abrigo da luz, imediatamente após as amostragens.

\section{Determinação gravimétrica}

A determinação gravimétrica do material particulado foi realizada pesando os filtros antes e após a coleta em ultra balança analítica com precisão de 1,0 $\mu$ g (METTLER TOLEDO AX26, Suíça). Os filtros foram inicialmente mantidos em estufa (Biopar - S150ST, Brasil) a $90{ }^{\circ} \mathrm{C}$ por 24 horas e então armazenados em placas de Petri em dessecador contendo sílica gel, seguidos por ambientação em sala de pesagem por mais 24 horas à temperatura e umidade relativa controladas de $20^{\circ} \mathrm{C}$ e $50 \%$ ( $\pm 5 \%$ ), respectivamente. Após coleta, os filtros foram submetidos as mesmas condições de armazenamento e ambientação antes das pesagens. ${ }^{33}$

\section{Medidas de reflectância para determinação de Black Carbon}

Após a determinação gravimétrica, foi realizada análise de reflectância no EEL 43D Smoke Stain Reflectometer (Diffusion Systems $L t d$, Reino Unido). Foi conferida a linearidade da medida de reflectância pelo ajuste do "zero" com o sensor desconectado da central, seguido do ajuste do " 100 " pela medida de um filtro branco fornecido com o equipamento. A reflectância da amostra foi tomada como a média de cinco medidas (uma central e quatro nas extremidades do filtro) ${ }^{34} \mathrm{~A}$ partir dos valores de reflectância, área do filtro e volume de ar amostrado foi calculada a concentração de BC na amostra. ${ }^{35}$

\section{HPA: extração e determinação cromatográfica}

Filtros e cartuchos foram submetidos à extração por agitação ultrassônica (THORNTON ${ }^{\circledR}$ modelo T14, Brasil) durante 45 min com $30 \mathrm{~mL}$ de acetonitrila (J. T. Baker, Estados Unidos) e metanol (J. T. Baker, Estados Unidos) (1:1 v/v). Os extratos foram concentrados por microdestilação à $65^{\circ} \mathrm{C}$ e o volume final foi completado com acetonitrila até $2 \mathrm{~mL}$. $\mathrm{O}$ extrato foi analisado por cromatografia líquida de alta eficiência no cromatógrafo modelo Ultimate 3000 (DAD/FLU) (Dionex, EUA) com coluna MetaSil ODS (METACHEM ${ }^{\circledR}$, Estados Unidos) (250 x 4,6 mm, $5 \mu \mathrm{m})$, injeção automática com alça de $20 \mu \mathrm{L}$ e fase móvel com gradiente de programação $\mathrm{H}_{2} \mathrm{O} / \mathrm{CH}_{3} \mathrm{CN}$ com porcentagem de $\mathrm{CH}_{3} \mathrm{CN}(35,65,100$ e 100$) \%$ à $(0,2,16$ e 18) min, respectivamente, à vazão de $1,7 \mathrm{~mL} \mathrm{~min}^{-1}$. Os HPA analisados foram: naftaleno (NAF), acenaftileno (ACE), acenafteno (ACF), fluoreno (FLU), fenantreno (FEN), antraceno (ANT), fluoranteno (FOR), pireno (PIR), benzo(a)antraceno (BAA), criseno (CRI), benzo(b)fluoranteno (BBF), benzo(k)fluoranteno (BKF), benzo(a) pireno (BAP), dibenzo(a,h)antraceno (DBA), indeno(1,2,3-cd)pireno (IND) e benzo(g,h,i)perileno (BGP). ${ }^{36,37}$ As concentrações de HPA foram obtidas por comparação com padrão (SUPELCO PAH Calibration Mix 4-7940-U, EUA). Curvas analíticas foram obtidas por triplicata de 10 a $500 \mathrm{ng} \mathrm{mL}^{-1}$ de HPA, obtendo coeficientes $\mathrm{R}^{2} \geq 0,99$. A quantificação foi realizada através dos cromatogramas de fluorescência com excitação molecular em 254 nm e emissão no modo zero order (onde a grade monocromadora serve como espelho e todo o espectro de emissão é adquirido no canal). A única exceção foi o acenaftileno, o qual foi quantificado pelos cromatogramas de absorção molecular em $228 \mathrm{~nm}$, pois não fluorescem. Os limites de detecção (LD) e quantificação (LQ) para cada HPA foram obtidos por três e dez vezes o quociente do desvio padrão da menor concentração detectável pelo coeficiente angular da reta, respectivamente e são apresentados na Tabela $2 .{ }^{38}$ Brancos de campo foram analisados com as amostras e a média das concentrações de cada HPA foi subtraída das concentrações finais nas amostras. A porcentagem de recuperação pela extração foi conferida com padrão certificado. Por adição de volume conhecido de padrão de HPA $10 \mu \mathrm{g} \mathrm{mL}$ diretamente nos cartuchos ( $\mathrm{v}=20 \mu \mathrm{L}$ em triplicata) e nos filtros (v $=30 \mu \mathrm{L}$ em duplicata) foram realizados os testes de recuperação nas mesmas condições de extração e determinação cromatográfica dos cartuchos e filtros utilizados na amostragem. A porcentagem de recuperação é apresentada na Tabela 1. Foi aplicado o fator de correção na concentração final dos HPA nas amostras a partir das

Tabela 1. Porcentagem de recuperação de HPA obtida por teste em cartucho XAD e filtros (mesmas condições de extração das amostras) e limites de detecção e quantificação. Padrão certificado (SUPELCO PAH Calibration Mix 4-7940-U)

\begin{tabular}{|c|c|c|c|c|}
\hline \multirow{2}{*}{ HPA } & \multicolumn{2}{|c|}{$\%_{(\mathrm{m} / \mathrm{m})}$ Recuperação } & \multirow[b]{2}{*}{$\mathbf{L D}\left(\mathbf{n g} \mathbf{m L}^{-1}\right)$} & \multirow[b]{2}{*}{$\mathbf{L Q}\left(\mathbf{n g} \mathbf{~ m L}^{-1}\right)$} \\
\hline & XAD & Filtros & & \\
\hline NAF & 87 & & 1,37 & 4,15 \\
\hline $\mathrm{ACE}$ & 70 & & 4,47 & 13,55 \\
\hline ACF com FLU & 84 & & 0,43 & 1,32 \\
\hline FEN & 76 & & 1,32 & 3,99 \\
\hline ANT & 58 & 65 & 0,51 & 1,53 \\
\hline FOR & 76 & 79 & 1,09 & 3,30 \\
\hline PIR & 60 & 81 & 0,88 & 2,67 \\
\hline BAA com CRI & 53 & 92 & 0,50 & 1,53 \\
\hline $\mathrm{BBF}$ & 47 & 97 & 0,28 & 0,86 \\
\hline $\mathrm{BKF}$ & 44 & 97 & 0,28 & 0,86 \\
\hline BAP & 43 & 99 & 0,74 & 2,23 \\
\hline DBA & 41 & 101 & 2,62 & 7,95 \\
\hline IND & 35 & 103 & 0,51 & 1,54 \\
\hline BGP & 36 & 104 & 0,96 & 2,91 \\
\hline
\end{tabular}


porcentagens de recuperação de cada HPA. Por fim, a razão de diagnóstico (RD) em pares de HPA foi aplicada aos dados obtidos para obtenção das assinaturas das fontes de emissão.

\section{RESULTADOS E DISCUSSÃO}

\section{Material particulado e Black Carbon}

Os intervalos de concentração e as médias diárias do MP fino coletados durante obras na rodovia e no recapeamento do asfalto no interior do terminal estão apresentadas na Tabela 2. A comparação entre as médias nas duas faixas de tamanho mostra que $89 \%$ da massa de $\mathrm{MP}_{2,5}$ foi composta pela fração $\mathrm{MP}_{1,0}$ nas amostras obtidas na rodovia em obras e $73 \%$ na amostras obtidas no terminal.

Tabela 2. Intervalos de concentração e médias $\left(\mu \mathrm{g} \mathrm{m}^{-3}\right)$ de material particulado fino $\left(\mathrm{MP}_{2.5}\right.$ e $\left.\mathrm{MP}_{1,0}\right)$ e do Black Carbon coletados durante obras na rodovia e no interior do terminal de ônibus urbano em Londrina/PR

\begin{tabular}{lcc}
\hline Local & $\begin{array}{c}\text { Intervalo de concentração } \\
\text { e média do MP fino } \\
\left(\mu \mathrm{g} \mathrm{m}^{-3}\right)\end{array}$ & $\begin{array}{c}\text { Intervalo de concentração } \\
\text { e média de } \mathrm{BC} \text { no MP fino } \\
\left(\mu \mathrm{g} \mathrm{m}^{-3}\right)\end{array}$ \\
\hline Rodovia, $\mathrm{MP}_{1,0}$ & $5,6-20,5(11,6)$ & $2,0-6,8(3,6)$ \\
Rodovia, $\mathrm{MP}_{2,5}$ & $6,3-24,2(13,0)$ & $1,8-6,5(3,4)$ \\
Terminal, $\mathrm{MP}_{1,0}$ & $15,1-75,1(35,4)$ & $5,1-14,6(11,6)$ \\
Terminal, $\mathrm{MP}_{2,5}$ & $19,7-150(48,4)$ & $4,9-16,2(11,5)$ \\
\hline
\end{tabular}

A OMS estabeleceu diretrizes para concentrações de $\mathrm{MP}_{2,5}$ a longo e curto prazo de $10 \mu \mathrm{g} \mathrm{m}^{-3}$ (média anual) e $25 \mathrm{~g} \mathrm{~m}^{-3}$ (média de 24 horas). ${ }^{39}$ Os resultados na rodovia foram abaixo dos níveis estabelecidos pela OMS para curto prazo enquanto no terminal as concentrações ultrapassaram em até 6 vezes o valor recomendado de $25 \mu \mathrm{g} \mathrm{m}^{-3}$. Em 2008, em condições normais de operação no terminal, Martins et al. obtiveram concentrações de $\mathrm{MP}_{1,0}$ e $\mathrm{MP}_{2,5}$ entre 20 a $50 \mu \mathrm{g} \mathrm{m}^{-3},{ }^{40}$ assim como os sete últimos dias de coleta, correspondentes à operação normal no terminal. Nos dois primeiros dias de coleta houve grande contribuição de MP fino proveniente da reforma do asfalto com concentrações de $\mathrm{MP}_{1,0}$ e $\mathrm{MP}_{2,5}$ entre de $70 \mathrm{e}$ $150 \mu \mathrm{g} \mathrm{m}^{-3}$. $\mathrm{O} \mathrm{MP}_{2,5}$ pode penetrar nos pulmões e causar vários tipos de doenças. O risco de mortalidade por doenças cardiopulmonares aumenta em $6 \%$ e o risco de mortalidade por câncer de pulmão em $8 \%$ com o aumento da concentração de MP em $10 \mu \mathrm{g} \mathrm{m}^{-3} .{ }^{41}$

Concentrações de BC obtidas no MP fino no ambiente aberto (PR-445) mostraram pouca variação comparando as faixas $\mathrm{MP}_{1,0} \mathrm{e}$ $\mathrm{MP}_{2,5}$. No interior do terminal, as concentrações de $\mathrm{BC}$ apresentaram valores em torno de $300 \%$ maiores do que as amostras obtidas em ar externo. O BC é possivelmente cancerígeno para os seres humanos (Grupo 2B), mas os limites de exposição ocupacional de BC no ar em vários países são em mg por metro cúbico, portanto está acima das concentrações de BC obtidas para obras de pavimentação asfáltica, conforme a Tabela 3. No Brasil e nos Estados Unidos a exposição ocupacional à $\mathrm{BC}$ em turno de 8 horas é de $3,5 \mathrm{mg} \mathrm{m}^{-3}$, assim como recomendado pelo NIOSH e pela OSHA. ${ }^{42}$

A Figura 2 apresenta a variação do $\mathrm{BC}$ na fração fina do MP coletados durante a reconstrução da rodovia, incluindo dados meteorológicos como temperatura, taxa de precipitação pluviométrica e velocidade dos ventos. A ocorrência de pouca precipitação durante o período de amostragem e pouca variação da velocidade dos ventos não interferiram no perfil de concentração do $\mathrm{BC}$ nas frações do MP fino. A Figura 3 apresenta a variação do $\mathrm{BC}$ na fração fina do MP coletados durante a reforma do piso asfáltico no interior do terminal urbano de Londrina.

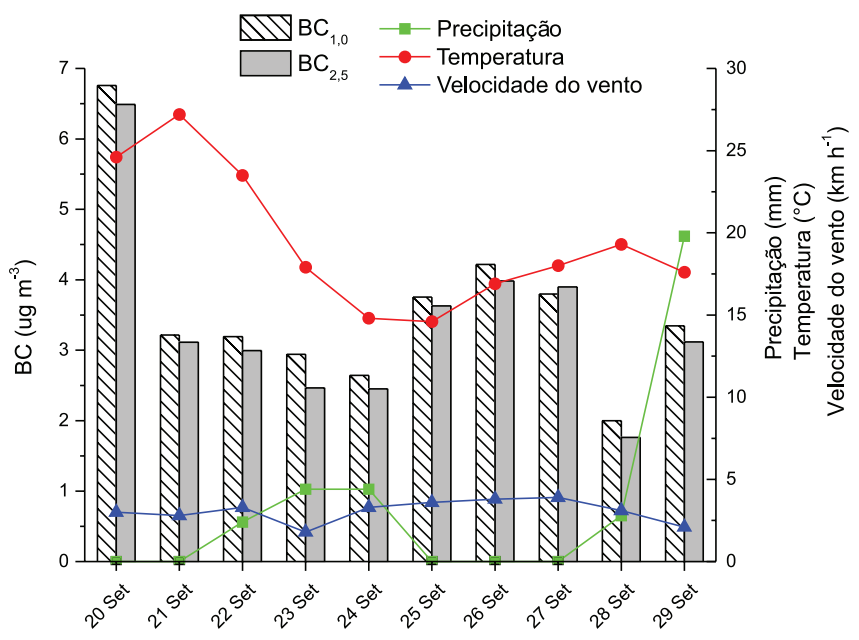

Figura 2. Eixo à direita: precipitação $(\mathrm{mm})$, temperatura $\left({ }^{\circ} \mathrm{C}\right)$, velocidade dos ventos $\left(\mathrm{km} \mathrm{h}^{-1}\right)$; eixo à esquerda: concentração diária de $B C\left(\mu \mathrm{g} \mathrm{\textrm {m } ^ { - 3 } )}\right.$ no material particulado fino $\left(M P_{1,0}\right.$ e $\left.M P_{2,5}\right)$ durante reconstrução da $P R-445$ no período de 20 a 29 de setembro de 2013, Londrina

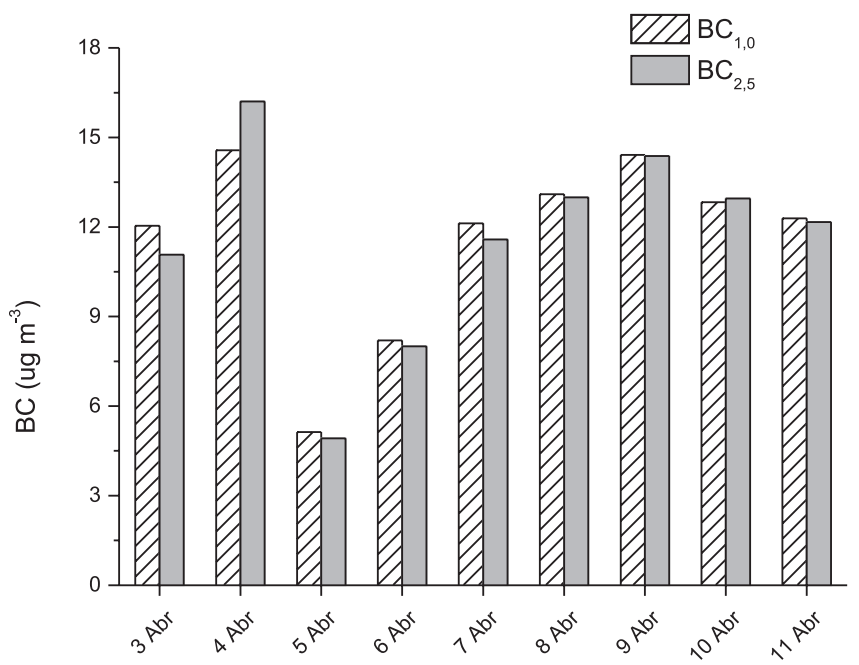

Figura 3. Concentração de $B C\left(\mu \mathrm{g} \mathrm{m}^{-3}\right)$ no material particulado fino $\left(M P_{1,0} e\right.$ $\left.M P_{2,5}\right)$ durante obras de reforma no asfalto do terminal urbano de Londrina no período de 03 a 11 de abril de 2014

Os três primeiros dias de amostragem no interior do terminal compreenderam atividades de obras no asfalto. Assim como na PR-445 (ambiente aberto), os valores de BC obtidas no terminal mostraram pouca variação. $\mathrm{O}$ declive na concentração de $\mathrm{BC}$ no dia 05 de abril de 2014 pode ser explicado por ser o último dia de obras apenas com reparos no asfalto e com os ônibus ainda fora de operação. A retomada da operação normal dos ônibus foi acompanhada pelo aumento gradativo na concentração de BC.

\section{Hidrocarbonetos policíclicos aromáticos (HPA)}

Durante as obras de pavimentação da rodovia as concentrações de HPA na fase gasosa (FG) foram maiores que as determinadas no MP com predominância dos HPA de baixa massa molar (Tabela 3). Na FG, naftaleno, acenafteno com fluoreno excederam a concentração de $330 \mathrm{ng} \mathrm{m}^{-3}$ em todos os dias de coleta. Fenantreno ultrapassou 46,0 $\mathrm{ng} \mathrm{m}^{-3}$; acenaftileno excedeu $125 \mathrm{ng} \mathrm{m}^{-3} \mathrm{em} 5$ dos dias de campanha além de outros HPA. Na fase particulada, naftaleno e acenaftileno atingiram concentrações máximas de 29,2 e $17,7 \mathrm{ng} \mathrm{m}^{-3}$ na faixa $\mathrm{MP}_{1,0}$, e 14,5 e $15,3 \mathrm{ng} \mathrm{m}^{-3}$ na faixa $\mathrm{MP}_{2,5}$, respectivamente, durante os trabalhos de duplicação da rodovia, 
Tabela 3. Massa molar (g) de HPA. Concentrações diárias $\left(\mathrm{ng} \mathrm{m}^{-3}\right)$ mínima, média e máxima de HPA e $\Sigma \mathrm{HPA}$ no material particulado fino $\left(\mathrm{MP}_{1,0}\right.$ e $\left.\mathrm{MP}_{2,5}\right)$ e na fase gasosa. Reconstrução da PR-445. Entre 20 e 29 de setembro de 2013

\begin{tabular}{|c|c|c|c|c|c|c|c|c|c|c|}
\hline \multirow{2}{*}{$\begin{array}{l}\text { HPA } \\
\left(\mathrm{ng} \mathrm{m}^{-3}\right)\end{array}$} & \multirow{2}{*}{$\begin{array}{c}\text { Massa } \\
\text { molar }(\mathrm{g})\end{array}$} & \multicolumn{3}{|c|}{ FG } & \multicolumn{3}{|c|}{$\mathrm{MP}_{1,0}$} & \multicolumn{3}{|c|}{$\mathrm{MP}_{2,5}$} \\
\hline & & Min. & Med. & Max. & Min. & Med. & Max. & Min. & Med. & Max \\
\hline NAF & 128,17 & 335 & 436 & 523 & ND & 8,77 & 29,2 & ND & 8,46 & 14,5 \\
\hline ACE & 152,20 & 125 & 234 & 277 & ND & 15,1 & 17,7 & ND & 9,47 & 15,3 \\
\hline ACF com FLU & $\begin{array}{l}154,21 \\
166,22\end{array}$ & 347 & 435 & 521 & 0,66 & 0,82 & 1,26 & 0,63 & 0,96 & 1,40 \\
\hline FEN & 178,23 & 46,0 & 138 & 296 & ND & 1,96 & 4,79 & ND & 0,63 & 0,98 \\
\hline ANT & 178,23 & 16,8 & 20,8 & 32,7 & 1,34 & 1,45 & 1,56 & 1,31 & 1,38 & 1,44 \\
\hline FOR & 202,26 & 3,55 & 4,40 & 5,34 & $\mathrm{NQ}$ & 1,27 & 3,83 & 0,38 & 0,76 & 1,74 \\
\hline PIR & 202,26 & 6,96 & 14,1 & 27,3 & 1,18 & 1,62 & 2,98 & 1,09 & 1,46 & 1,96 \\
\hline BAA com CRI & $\begin{array}{l}228,29 \\
228,29\end{array}$ & 12,3 & 17,3 & 21,7 & 1,10 & 1,25 & 1,49 & 1,08 & 1,22 & 1,36 \\
\hline BBF & 252,32 & 14,8 & 22,0 & 27,2 & 1,03 & 1,19 & 1,40 & 1,08 & 1,20 & 1,51 \\
\hline BKF & 252,32 & 15,3 & 21,3 & 25,4 & 0,98 & 1,12 & 1,23 & 1,02 & 1,10 & 1,23 \\
\hline BAP & 252,32 & 15,3 & 20,9 & 25,4 & 0,93 & 1,08 & 1,27 & 1,01 & 1,06 & 1,18 \\
\hline DBA & 278,35 & 17,0 & 22,7 & 33,4 & 0,85 & 0,89 & 0,96 & 0,78 & 0,98 & 1,84 \\
\hline IND & 276,34 & 25,1 & 34,7 & 40,3 & 1,20 & 1,39 & 1,62 & 1,28 & 1,38 & 1,49 \\
\hline BGP & 276,34 & 26,2 & 34,9 & 40,9 & 1,26 & 1,47 & 1,87 & 1,32 & 1,48 & 1,67 \\
\hline$\Sigma$ LPA & & 937 & 1337 & 1704 & 12,1 & 25,6 & 52,1 & 11,5 & 21,9 & 37,0 \\
\hline
\end{tabular}

$\mathrm{ND}=$ não detectado. $\mathrm{NQ}$ = não quantificado. $\mathrm{FG}$ = fase gasosa. Determinação simultanea de ACF com FLU e BAA com CRI.

além de outros HPA identificados. Os perfis obtidos de emissão dos 16 HPAs encontram-se na Figura 4.

Naftaleno, acenaftileno, fenantreno e fluoranteno atingiram a concentração máxima de $1642,765,485$ e $164 \mathrm{ng} \mathrm{m}^{-3}$, respectivamente, nos dois primeiros dias de coleta no interior do terminal e foram os únicos HPA identificados na FG (Tabela 4). Tavares et al. determinaram HPA na FG no mesmo local, em condições normais de operação do terminal, relatando concentrações máximas de naftaleno (110 $\left.\mathrm{ng} \mathrm{m}^{-3}\right)$, acenaftileno (92 $\left.\mathrm{ng} \mathrm{m}^{-3}\right)$, fenantreno (389 $\left.\mathrm{ng} \mathrm{m}^{-3}\right)$, fluoranteno (48 $\left.\mathrm{ng} \mathrm{m}^{-3}\right)$, acenafteno $\left(70 \mathrm{ng} \mathrm{m}^{-3}\right)$, fluoreno (159 $\left.\mathrm{ng} \mathrm{m}^{-3}\right)$, antraceno $\left(39 \mathrm{ng} \mathrm{m}^{-3}\right)$, pireno $\left(52 \mathrm{ng} \mathrm{m}^{-3}\right)$, benzo(a)antraceno $\left(2 \mathrm{ng} \mathrm{m}^{-3}\right)$ e criseno $\left(5 \mathrm{ng} \mathrm{m}^{-3}\right) \cdot{ }^{36} \mathrm{~A}$ comparação dos dois resultados mostra diferentes perfis de emissão para cada ocasião e faixas de concentração bem mais elevadas durante a aplicação do asfalto no local.

Durante a reforma do terminal, os HPA de 4 anéis foram os de concentração mais elevada na fração particulada. Benzo(b) fluoranteno, pireno e benzo(a)antraceno com criseno atingiram concentrações de $137,47,1$ e $41,0 \mathrm{ng} \mathrm{m}^{-3}$, respectivamente, nos dois primeiros dias de coleta, além de outros HPA identificados (Tabela 4 e Figura 5). Martins et al. determinaram HPA em MP no terminal em condições normais de operação e não detectaram HPA leves (2 e 3 anéis). Comparando a faixa de concentração dos HPA com os resultados de Martins et al. (máxima de $1 \mathrm{ng} \mathrm{m}^{-3}$ ), observa-se que os níveis obtidos nos sete últimos dias de campanha (Figura 5), correspondente à operação normal do terminal. ${ }^{40}$ A comparação confirma a contribuição de HPA no MP fino proveniente da reforma no asfalto do terminal.

As concentrações de HPA no ar ambiente do terminal representam a distribuição de HPA entre as fases gasosa e particulada. Na Tabela 5 observa-se os HPA mais leves que foram quantificados na fase vapor e os HPA mais pesados quantificados na fase particulada. Durante as duas ocasiões de atividades laborais impactadas com emissão asfáltica, os resultados da campanha no interior do terminal representam um ambiente de fonte exclusiva enquanto à margem da rodovia, sendo o ambiente aberto, houve a possibilidade de contribuição de outras fontes de emissão.

Tang e Isacsson caracterizaram a emissão de óleo asfáltico de petróleo atribuindo-a predominantemente ao fenantreno. ${ }^{43}$ Pelos resultados do nosso trabalho, a emissão atmosférica de HPA proveniente da aplicação de derivado asfáltico em obras de pavimentação foi atribuída à classe dos HPA mais leves, como proposto por Wang et al. em condições de pavimentação simuladas em laboratório. ${ }^{6}$ Ou ainda, mais especificamente aos de dois anéis, como proposto por Lee et al. na emissão de fumos de asfalto no preparo industrial. ${ }^{2}$ Nas amostragens na rodovia, os HPA leves corresponderam a $85 \%$ dos HPA totais enquanto no terminal, 93\% dos HPA determinados corresponderam aos HPA leves.

$\mathrm{Na}$ campanha realizada no terminal, a reforma no asfalto aconteceu apenas nos três primeiros dias, assim, devido à contribuição de emissão atmosférica proveniente da pavimentação asfáltica para os compostos presentes no ar, é possível observar uma tendência decrescente nos valores de concentração de HPA no decorrer dos dias na Figura 5. Essa tendência não pode ser observada para a rodovia na Figura 4, pois, nesse caso, do primeiro ao último dia de campanha houve aplicação de derivado asfáltico.

Durante as obras na rodovia e no terminal as concentrações foram próximas ao nível recomendado de $2 \mathrm{ng} \mathrm{m}^{-3}$ para benzo(a)pireno, assim como a detecção de dibenzo(a,h)antraceno que, de acordo com a US-EPA, possui o mesmo potencial carcinogêneo do benzo(a) pireno. ${ }^{26,27}$ Concentrações de benzo(a)pireno superiores de $1,2 \mathrm{ng} \mathrm{m}^{-3}$ (WHO, risco de câncer de 1/10000) foram obtidas tanto nas obras da rodovia quanto no terminal. ${ }^{30} \mathrm{O}$ IARC considera o benzo(a)pireno como carcinogêneo e benzo(a)antraceno e criseno possivelmente cancerígenos. Benzo(a)antraceno com criseno foram identificados nos dois locais de coleta e atingiram concentração de até $41,0 \mathrm{ng} \mathrm{m}^{-3}$ no MP fino no terminal. ${ }^{25}$ 

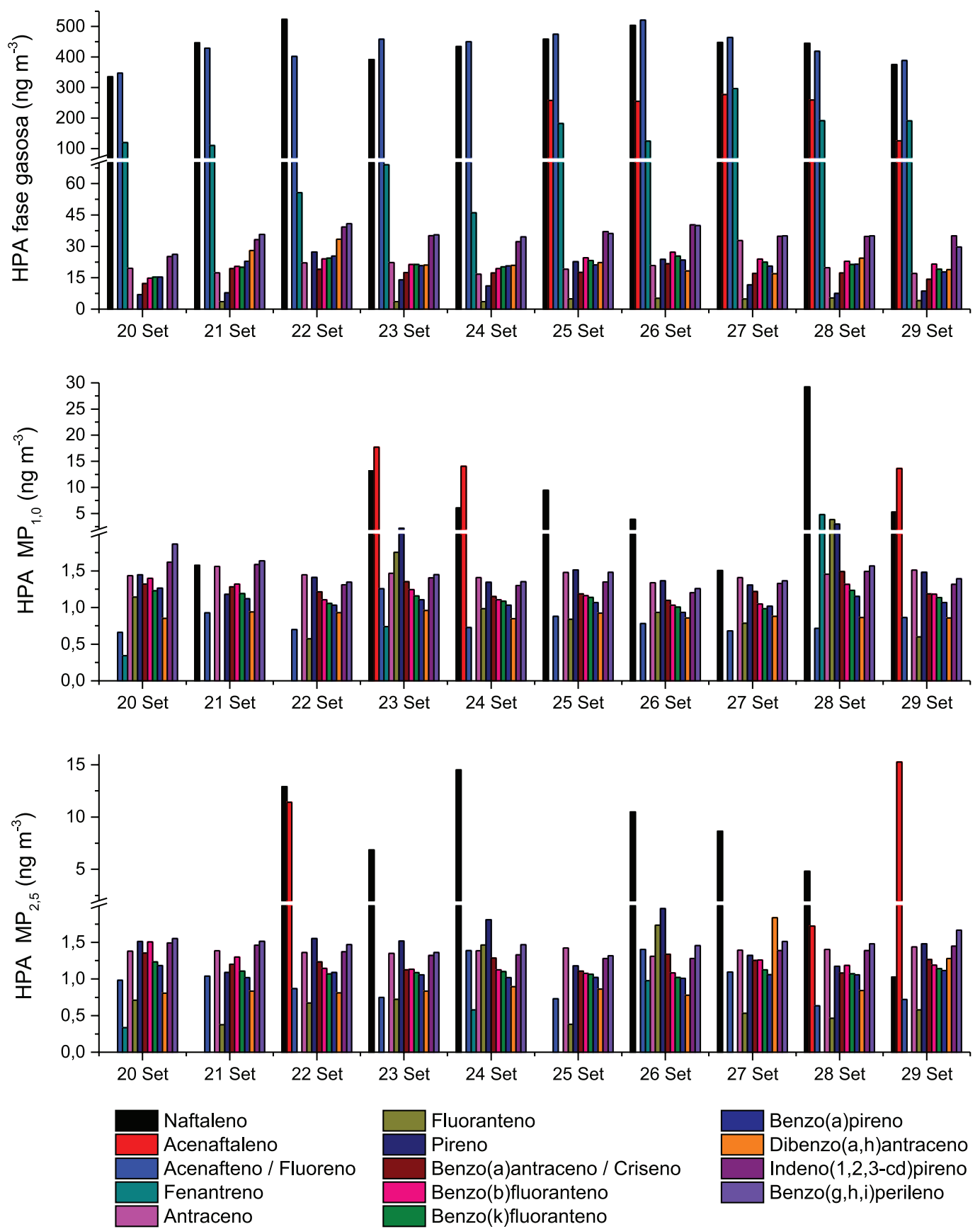

Figura 4. Concentração de HPA (ng $\left.\mathrm{m}^{-3}\right)$ na fase gasosa e no $M P$ fino $\left(M P_{1,0}\right.$ e $\left.M P_{2,5}\right)$ durante obras de reconstrução da rodovia no período de 20 a 29 de setembro de 2013, Londrina, Paraná

Naftaleno apresentou a concentração mais alta entre os HPA com valores de 523 e $1642 \mathrm{ng} \mathrm{m}^{-3}$ na rodovia e no terminal, respectivamente. De acordo com o CDC naftaleno no ar pode irritar os olhos e as vias respiratórias. ${ }^{24}$

Os intervalos de concentração das somatórias $\Sigma$ HPA nas fases gasosa, $\mathrm{MP}_{1,0}$ e $\mathrm{MP}_{2,5}$ foram respectivamente, 937-1704; 12,1-52,1 e $11,5-37,0 \mathrm{ng} \mathrm{m}^{-3}$ na reconstrução da rodovia e no interior do terminal de ônibus foram 136-2794; 3,0-183 e 1,6-194 ng m ${ }^{-3}$ na FG, $\mathrm{MP}_{1,0}$ e $\mathrm{MP}_{2,5}$, respectivamente. Esses valores podem ser comparados a recomendações da NIOSH e da OSHA. A NIOSH atribui o nível de exposição de $100 \mathrm{ng} \mathrm{m}^{-3}$ a voláteis de petróleo para ambiente laboral em turno de 8 horas e a OSHA considera o nível de exposição ao ar no ambiente de trabalho com $200 \mathrm{ng} \mathrm{m}^{-3}$ de fração de petróleo solúvel em benzeno. ${ }^{30,31}$ Portanto, os trabalhadores nos dois ambientes estiveram impactados à elevadas concentrações de HPA.

\section{Razões de diagnóstico (RD)}

A classificação do tipo de fonte de emissão dos HPA é atribuída de acordo com as bases do cálculo utilizado na razão de diagnóstico. As taxas de HPA calculadas para cada fonte hipotética não são definitivas: por exemplo, a taxa de diagnóstico relatada pode mostrar fortes variações para uma fonte específica. Dada a complexidade dos processos envolvidos, essa é uma suposição mais arriscada e para permitir identificar melhor as fontes, geralmente são usadas taxas combinadas. Similaridades são encontradas entre as fontes, assim como mais de uma fonte pode ser atribuida. ${ }^{21,32}$

Considera-se a fonte de emissão de HPA como mista para os dois ambientes onde ocorreram as amostragens. Na duplicação da rodovia PR-445 considerando os 16 HPA analisados, as razões entre os HPA menores ( 2 e 3 anéis) e maiores (4 a 6 anéis) foram superiores a 1 
Tabela 4. Massa molar (g) de HPA. Concentrações diárias (ng m³ ${ }^{-3}$ mínima, média e máxima de HPA e $\Sigma$ HPA no material particulado fino $\left(\mathrm{MP}_{1,0}\right.$ e $\left.\mathrm{MP}_{2,5}\right)$ e na fase gasosa (FG). Obra de reforma no terminal de ônibus urbano de Londrina. Entre 3 e 11 de abril de 2014

\begin{tabular}{|c|c|c|c|c|c|c|c|c|c|c|}
\hline \multirow{2}{*}{$\begin{array}{l}\text { HPA } \\
\left(\mathrm{ng} \mathrm{m}^{-3}\right)\end{array}$} & \multirow{2}{*}{$\begin{array}{c}\text { Massa } \\
\text { molar }(\mathrm{g})\end{array}$} & \multicolumn{3}{|c|}{ FG } & \multicolumn{3}{|c|}{$\mathrm{MP}_{1,0}$} & \multicolumn{3}{|c|}{$\mathrm{MP}_{2,5}$} \\
\hline & & Min. & Med. & Max. & Min. & Med. & Max. & Min. & Med. & Max. \\
\hline NAF & 128,17 & ND & 400 & 1642 & ND & - & - & ND & - & - \\
\hline ACE & 152,20 & 85,9 & 379 & 765 & ND & - & - & ND & - & - \\
\hline ACF com FLU & $\begin{array}{l}154,21 \\
166,22\end{array}$ & ND & - & - & ND & - & - & ND & - & - \\
\hline FEN & 178,23 & ND & 118 & 485 & ND & - & - & ND & - & - \\
\hline ANT & 178,23 & ND & - & - & ND & - & - & ND & - & - \\
\hline FOR & 202,26 & ND & 23,0 & 164 & ND & 5,89 & 34,8 & ND & 0,96 & 3,14 \\
\hline PIR & 202,26 & ND & - & - & ND & 8,95 & 47,1 & ND & 4,42 & 17,5 \\
\hline BAA com CRI & $\begin{array}{l}228,29 \\
228,29\end{array}$ & ND & - & - & 1,29 & 8,53 & 29,7 & ND & 5,98 & 41,0 \\
\hline BBF & 252,32 & ND & - & - & ND & 10,1 & 89,5 & ND & 30,8 & 137 \\
\hline BKF & 252,32 & ND & - & - & ND & - & - & ND & 0,08 & 0,73 \\
\hline BAP & 252,32 & ND & - & - & ND & 0,37 & 1,33 & ND & 0,46 & 1,39 \\
\hline DBA & 278,35 & ND & - & - & ND & - & - & ND & - & - \\
\hline IND & 276,34 & ND & - & - & ND & 0,36 & 3,25 & ND & 0,95 & 4,33 \\
\hline BGP & 276,34 & ND & - & - & ND & - & - & ND & - & - \\
\hline$\Sigma$ LHPA & & 136 & 919 & 2794 & 3,01 & 34,3 & 183 & 1,59 & 43,7 & 194 \\
\hline
\end{tabular}

$\mathrm{ND}$ = não detectado. $\mathrm{NQ}$ = não quantificado. $\mathrm{FG}$ = fase gasosa. - = não aplicável. Determinação simultanea de ACF com FLU e BAA com CRI.
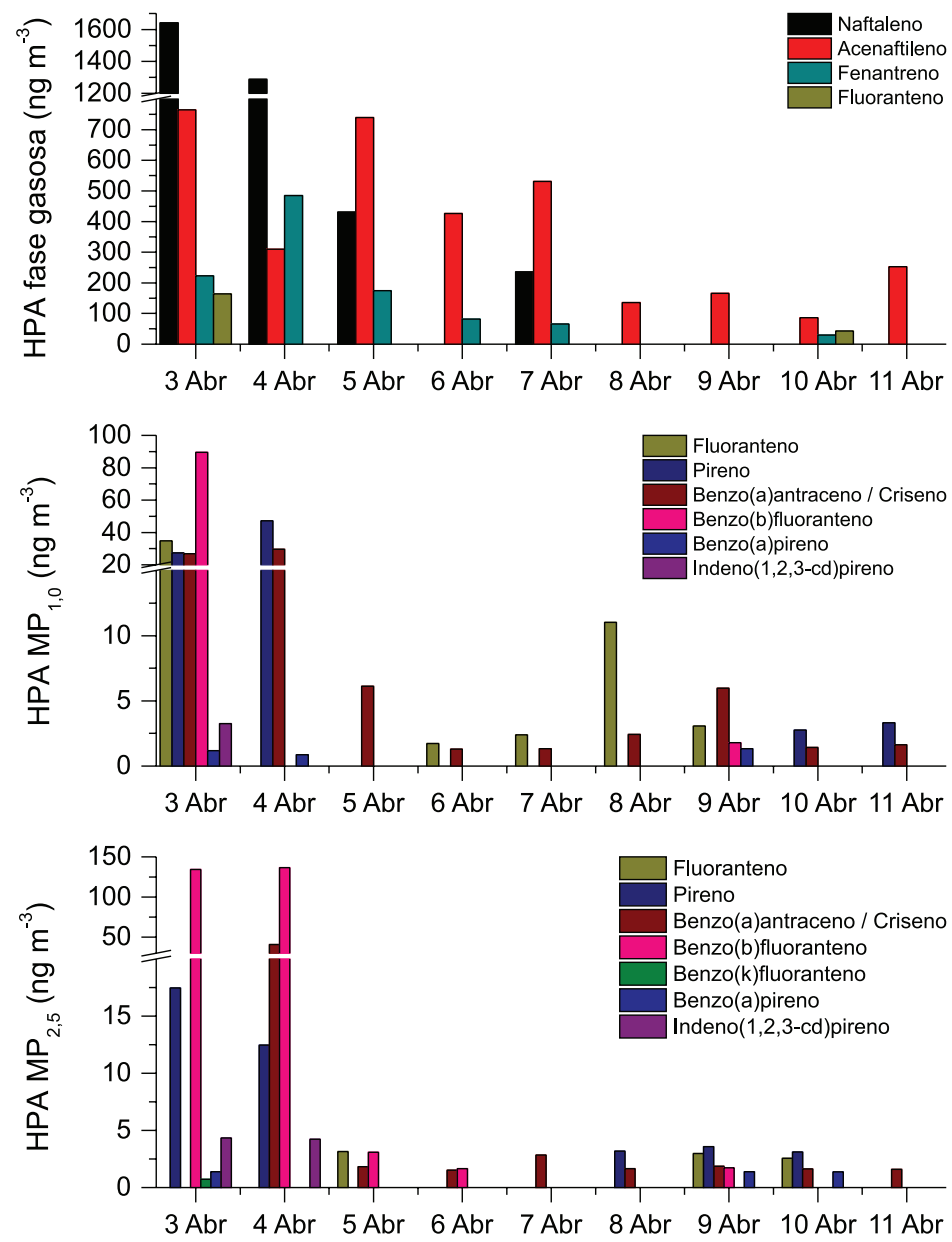

Figura 5. Concentração de HPA (ng $\left.\mathrm{m}^{-3}\right)$ em fase gasosa e particulada $\left(\mathrm{MP}_{1,0}\right.$ e $\left.M P_{2,5}\right)$. Obra de reforma no asfalto do terminal urbano de Londrina. Período 03 a 11 de abril de 2014 
Tabela 5. Razão de diagnóstico entre HPA nas fases gasosa e particulada $\left(\mathrm{MP}_{1,0} \mathrm{e} \mathrm{MP}_{2.5}\right)$ durante amostragem em condições reais de aplicação asfáltica. Obras de duplicação de rodovia e recapeamento asfáltico no interior do terminal urbano de Londrina. Atribuição da fonte de acordo com a RD calculada e a faixa respectiva

\begin{tabular}{|c|c|c|c|c|c|c|c|c|}
\hline \multirow{2}{*}{ Razão diagnóstico } & \multirow{2}{*}{ Faixa } & \multirow{2}{*}{ Fonte } & \multicolumn{3}{|c|}{ Duplicação da rodovia } & \multicolumn{3}{|c|}{ Terminal urbano } \\
\hline & & & FG & $\mathrm{MP}_{1,0}$ & $\mathrm{MP}_{2,5}$ & FG & $\mathrm{MP}_{1,0}$ & $\mathrm{MP}_{2,5}$ \\
\hline$A N T$ & $<0,1$ & Petrogênica & 0,1 & & & n.a & n.a & n.a \\
\hline$\overline{A N T+F E N}$ & $>0,1$ & Pirogênica & & 0,7 & 0,9 & & & \\
\hline FEN & $<10$ & Pirogênica & 6,7 & 0,4 & 0,1 & n.a & n.a & n.a \\
\hline$\overline{A N T}$ & $>10$ & Petrogênica & & & & & & \\
\hline FOR & $<1$ & Petrogênica & 0,25 & 0,70 & 0,52 & $\mathrm{n} . \mathrm{a}$ & 0,47 & n.a \\
\hline$\overline{P I R}$ & $>1$ & Pirogênica & & & & & & \\
\hline \multirow{3}{*}{$\frac{F O R}{F O R+P I R}$} & $<0,4$ & Petrogênica & 0,20 & & 0,34 & n.a & 0,32 & n.a \\
\hline & $(0,4-0,5)$ & CFL & & 0,41 & & & & \\
\hline & $>0,5$ & Pirogênica & & & & & & \\
\hline \multirow{3}{*}{$\frac{I N D}{I N D+B G P}$} & $<0,2$ & Petrogênica & & & & $\mathrm{n} . \mathrm{a}$ & n.a & n.a \\
\hline & $(0,2-0,5)$ & CFL & 0,50 & 0,49 & 0,48 & & & \\
\hline & $>0,5$ & Pirogênica & & & & & & \\
\hline$\sum H P A 2$ e 3 anéis & $<1$ & Pirogênica & & & & & 0,15 & n.a \\
\hline$\sum H P A 4$ e 6 anéis & $>1$ & Petrogênica & 6,20 & 1,55 & 1,22 & $>1$ & & \\
\hline
\end{tabular}

FG = fase gasosa; $\mathrm{CFL}=$ combustível fóssil líquido; n.a. = não aplicável. Adaptado das referências 21 e 32.

em todas as frações mostrando a contribuição petrogênica. A razão fluoranteno/pireno menor que 1 em todas as frações foi associada a fontes petrogênicas, no entanto, a razão fenantreno/antraceno menor que 10 é de origem pirogênica, apesar de forte contribuição petrogênica na fase gasosa (RD 6,7). A razão antraceno/(fenantreno + antraceno) atribui o diagnóstico como contribuições de fontes mistas petrogênica $(\mathrm{FG})$ e pirogênica $(\mathrm{MP})$. $\mathrm{O}$ diagnóstico obtido à partir da razão fluoranteno/(fluoranteno + pireno) aponta como origem petrogênica além da contribuição de fontes de queima de combustível fóssil líquido. As razões indeno/(indeno + benzo(g,h,i)perileno) nas FG e MP revelam a contribuição de combustível fóssil líquido. Dessa forma, foi possível atribuir que os HPA encontrados na FG e MP foram principalmente de origem petrogênica com contribuição pirogênica incluindo a queima de combustíveis fósseis líquidos. As atribuições se justificam ao considerar: i) emissões provenientes da aplicação de camada selante em pista de derivado asfáltico, ii) emissões provenientes dos escapamentos dos veículos em tráfego na marginal a rodovia e iii) queimadas irregulares nos arredores do local de coleta.

No cálculo RD na reforma do asfalto no terminal foram considerados apenas os dois primeiros dias de coleta, a fim de selecionar exclusivamente emissões provenientes da reforma e não daquelas provenientes dos ônibus em circulação. A razão entre os HPA mais leves e mais pesados no $\mathrm{MP}_{1,0}$ atribui como fonte pirogênica enquanto as razões fluoranteno/(fluoranteno + pireno) e fluoranteno/pireno indicam fontes petrogênicas no teminal e seu entorno. Os HPA na fase gasosa também são considerados de fonte mista petrogênica e pirogênica, no entanto, a contribuição petrogênica foi predominante pois os HPA de 2 e 3 anéis foram abundantes enquanto os de 4 a 6 anéis não foram detectados.

$\mathrm{O}$ ambiente semiaberto no terminal propicia menor influência de outras fontes de emissão, assim como menor influência de fatores como diluição dos poluentes provocada por ventos e a degradação de HPA por incidência de luz. Em geral, os resultados mostraram as assinaturas que caracterizam a formação de HPA a partir de processos pirogênicos, por evaporação de fontes petrogênicas e queima de combustíveis fósseis líquidos, considerando as amostragens realizadas em condições reais de operação, reforçando a caracterização das fontes de emissão por RD..$^{44,45}$

\section{CONCLUSÃO}

A emissão de MP inalável fino proveniente de pavimentação asfáltica na reforma do terminal está acima dos limites recomendados pela OMS sendo preocupante quanto à saúde ocupacional.

O MP fino possui maior potencial de aquecimento global devido ao elevado teor de BC, portanto, considerando as alterações climáticas, os níveis de concentração de BC obtidos para as assinaturas durante pavimentação asfáltica são de interesse ambiental.

A aplicação de derivados asfálticos como fonte petrogênica é responsável pela emissão de HPA de massa molares de 2 a 6 anéis, nas fases gasosa e particulada, sendo que os HPA de menor número de anéis são emitidos em concentração mais elevada. Durante a aplicação, compostos nocivos ao ambiente e à saúde do trabalhador são emitidos em alta concentração.

Espera-se que os resultados deste trabalho possam propiciar informações uteis à sociedade, fornecendo dados sobre potenciais contaminantes em circunstâncias específicas que vise beneficiar práticas de menor impacto ambiental e de maior proteção à saúde dos trabalhadores.

\section{AGRADECIMENTOS}

Os autores agradecem ao CNPq, à CAPES e à Fundação Araucária pelo fomento à pesquisa. Especialmente à CMTU pela permissão de utilização das dependências do terminal de ônibus urbano de Londrina, assim como ao Grêmio Esportivo Cacique Londrina, local onde foram colocados os amostradores à margem da rodovia. Ao Instituto Nacional de Ciência e Tecnologia de Energia e Ambiente (INCT - E\&A).

\section{REFERÊNCIAS}

1. Grzegorz, B.; Andrzej, P.; Marian, K.; Chemosphere 2014, 107, 23. 
2. Lee, W. J.; Chao, W. H.; Shih, M.; Tsai, C. H.; Chen, T. J. H.; Tsai, P. J.; Environ. Sci. Technol. 2004, 38, 5274.

3. Gasthauer, E.; Mazé, M.; Marchand, J. P.; Amouroux, J.; Fuel 2008, 87, 1428 .

4. http://www.inchem.org/documents/cicads/cicads/cicad59.htm, acessada em Março 2020.

5. Fernandes, P. R.; Soares S. A.; Nascimento, R. F.; Soares, J. B.; Cavalcante, R. M.; J. Chromatogr. Sci. 2009, 47, 789.

6. Wang, J.; Lewis, D. M.; Castranova, V.; Frazer, D. G.; Goldsmith, T.; Tomblyn, S.; Simpson, J.; Stone, S.; Afshari, A.; Siegel, P. D.; Anal. Chem. 2001, 73, 3691.

7. Tang, B.; Isacsson, U.; Fuel 2006, 85, 1232.

8. https://www.healthcouncil.nl/documents/advisory-reports/2007/02/13/ bitumen-vapour-and-aerosol, acessada em Março 2020.

9. https://www.cdc.gov/niosh/docs/2001-110/pdfs/2001-110.pdf, acessada em Março 2020.

10. Binet, S.; Pfohl-Leszkowicz, A.; Brandt, H.; Lafontaine, M.; Castegnaro, M.; Sci. Total Environ. 2002, 300, 37.

11. Schreiner, A, C.; Regul. Toxicol. Pharmacol. 2011, 59, 270.

12. Baird, C.; Cann, M.; Environmental chemistry, $5^{\text {th }}$ ed., Freeman and Company: New York, 2012.

13. Guarieiro, L. L. N.; Vasconcellos, P. C.; Solci, M. C.; Rev. Virtual Quim. 2011, 3, 434.

14. Wierzbicka, A.; Nilsson, P. T.; Rissler, J.; Sallsten, G.; Xu, Y.; Pagels, J. H.; Albin, M.; Österberg, K.; Strandberg, B.; Eriksson, A.; Bohgard, M.; Bergemalm-Rynell, K.; Gudmundsson, A.; Atmos. Environ. 2014, $86,212$.

15. Agarwal, T.; Thomas, B. D.; Environ. Pollut. 2011, 159, 64.

16. Bond, T. C.; Doherty, S. J.; Fahey, D. W.; Forster, P. M.; Berntsen, T.; Deangelo B. J.; Flanner, M. G.; Ghan, S.; Kärcher, B.; Koch, D.; Kinne, S.; Kondo, Y.; Quinn, P. K.; Sarofim, M. C.; Schultz, M. G.; Schulz, M.; Venkataraman, C.; Zhang, H.; Zhang, S.; Bellouin, N.; Guttikunda, S. K.; Hopke, P. K.; Jacobson, M. Z.; Kaiser, J. W.; Klimont, Z.; Lohmann, U.; Schwarz, J. P.; Shindell, D.; Storelvmo, T.; Warren, S. G.; Zender, C. S.; J. Geophys. Res.: Atmos. 2013, 118, 5380.

17. Pahalagedara, L.; Sharma, H.; Kuo, C. H.; Dharmarathna, S.; Joshi, A.; Suib, S. L.; Mhadeshwar, A. B.; Energy Fuels 2012, 26, 6757.

18. Corrêa, M. S.; Arbilla, G.; Atmos. Environ. 2006, 40, 6821.

19. https://onlinelibrary.wiley.com/doi/pdf/10.1002/3527600418. mb0223orge0027b, acessada em Março 2020.

20. Ravindra, K.; Sokhi, R.; Grieken, V. R.; Atmos. Environ. 2008, 42, 2895.

21. Tobiszewski, M.; Namiesnik, J.; Environ. Pollut. 2012, 162, 110.

22. Loeber, L.; Muller, G.; Morel, J.; Sutton, O.; Fuel 1998, 77, 1443.

23. Srogi, K.; Environ. Chem. Lett. 2007, 5, 169.
24. https://www.cdc.gov/biomonitoring/PAHs_FactSheet.html, acessada em Março 2020.

25. https://monographs.iarc.fr/list-of-classifications-volumes/, acessada em Março 2020.

26. https://www.epa.gov/sites/production/files/2015-11/documents/pah-rpfs. pdf, acessada em Março 2020.

27. https://cfpub.epa.gov/ncea/iris/iris_documents/documents/subst/0454_ summary.pdf, acessada em Março 2020.

28. https://cfpub.epa.gov/ncea/iris2/chemicalLanding.cfm?substance $\mathrm{nmbr}=456$, acessada em Março 2020.

29. https://cfpub.epa.gov/ncea/iris2/chemicallanding.cfm?substance_ nmbr=136, acessada em Março 2020.

30. http://www.euro.who.int/_data/assets/pdf_file/0005/74732/E71922.pdf, acessada em Março 2020.

31. https://www.atsdr.cdc.gov/csem/csem.asp?csem $=13 \& p o=8$, acessada em Março 2020.

32. Ratola, N.; Amigo, J. M.; Alves, A.; Arch. Environ. Contam. Toxicol. 2010, 58, 631.

33. Silva Jr., C. R.; Piracelli, V. P.; Sabino, F. C.; Amador, I. R.; Pinto, J. P.; Solci, M. C.; Rev. Virtual Quim. 2019, 11, 297.

34. http://uk-air.defra.gov.uk/assets/documents/reports/empire/smkman/ sch7.html, acessada em Março 2020

35. Silva Jr., C. R.; Lemos, B. R. L.; Pinto, J. P.; Amador, I. R.; Solci, M. C.; J. Braz. Chem. Soc. 2019, 30, 786.

36. Tavares Jr., M.; Pinto, J. P.; Souza, A. L.; Scarminio, I. S.; Solci, M. C.; Atmos. Environ. 2004, 38, 5039

37. https://www.cdc.gov/niosh/docs/2003-154/pdfs/5506.pdf, acessada em Março 2020.

38. Thompson, M.; Ellison, S. L. R.; Wood, R.; Pure Appl. Chem. 2002, 74, 835.

39. http://www.euro.who.int/_data/assets/pdf_file/0005/78638/E90038.pdf, acessada em Março 2020.

40. Martins, L. D.; Silva Jr., C. R.; Solci, M. C.; Pinto, J. P.; Souza, D. Z.; Vasconcellos, P.; Guarieiro, A. L. N.; Guarieiro, L. L. N.; Sousa, E.; Andrade, J. B.; Environ. Monit. Assess. 2012, 184, 2663.

41. Ianistcki, M.; Dallarosa, J.; Sauer, C.; Teixeira, C. E.; Silva, J.; Environ. Pollut. 2009, 157, 2037.

42. https://monographs.iarc.fr/wp-content/uploads/2018/06/mono93.pdf, acessada em Março 2020.

43. Tang, B.; Isacsson, U.; Fuel 2006, 85, 1232.

44. Haynes, B. S.; Wagner, H. G. G.; Prog. Energy Combust. Sci. 1981, 7 , 229.

45. Manaham, S. E.; Environmental chemistry, $7^{\text {th }}$ ed., Lewis Publishers: Washington, D. C., 2000. 